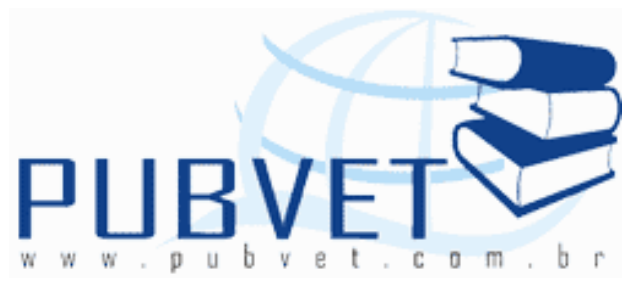

PUBVET, Publicações em Medicina Veterinária e Zootecnia.

\title{
Doença de Legg-Calvé-Perthes: Revisão bibliográfica
}

Marcos Vinícius Santana Filho ${ }^{1}$, Juan Benito Campos Diz Atan², Lucas Cristo Conilho Macedo Müller ${ }^{3}$, Christiano Costa Esposito ${ }^{4}$, Paulo César Silva ${ }^{5}$, Manoel Luiz Ferreira ${ }^{6}$

${ }^{1}$ Médico Veterinário Autônomo.

${ }^{2}$ Graduando em Medicina Veterinária da Universidade Federal Fluminense Niterói - RJ.

${ }^{3}$ Graduando em Medicina da Universidade Federal do Rio de Janeiro - Rio de Janeiro - RJ.

${ }^{4}$ Médico Veterinário. Pesquisador do Centro de Cirurgia Experimental da Universidade Federal do Rio de Janeiro (UFRJ) - Rio de Janeiro _ RJ.

${ }^{5}$ Professor Adjunto III da disciplina de Técnica Operatória da Faculdade de Medicina da Universidade Federal do Rio de Janeiro - Rio de Janeiro - RJ.

${ }^{6}$ Professor Assistente do Curso de Medicina Veterinária da Universidade Estadual de Santa Cruz - Ilhéus - BA.

\section{Resumo}

Este trabalho teve como objetivo rever a literatura referente a enfermidade de Legg-Calvé-Perthes. Doença conhecida por vários nomes: necrose asséptica ou avascular da cabeça femoral, osteocondrite deformante juvenil e coxa plana. Ocorre em cães entre 4 e 12 meses de idade, de raças pequenas quando existe necrose da cabeça e colo femoral devido a oclusão do suprimento sanguíneo. 
SANTANA FILHO, M.V. et al. Doença de Legg-Calvé-Perthes. Revisão bibliográfica. PUBVET, Londrina, V. 5, N. 9, Ed. 156, Art. 1052, 2011.

Os sinais clínicos consistem por claudicação gradual do membro afetado, dor e deformidade da articulação. O diagnóstico é realizado através do exame clínico e confirmado por radiografia. O tratamento para esta distrofia depende da severidade da condição podendo ser conservador ou cirúrgico.

Palavras-chave: Doença de Legg-Perthes. Osteonecrose. Necrose asséptica da cabeça do fêmur. Cabeça do fêmur.

\title{
Legg-Perthes Disease: Literature review
}

\begin{abstract}
Legg-Calvé-Perthes is a condition known by many names: aseptic or avascular necrosis of the femoral head, osteocondritis deformans juvenilis and coxa plana. It occours in young, small-breed dogs when there is necrosis of the femoral head and neck due to lack of blood supply. Typically clinical signs consist of lameness in one leg with deformity of the rip joint occouring in young dogs between 4 to 12 months old. The diagnosis is made by clinical signs and confirmed by radiographs. Treatment varies depending on the severity of the condition. The most of cases, pain is severe and surgical treatment is required. It consists is excision of the femoral head to form a pseudo-joint with good range of mobility.
\end{abstract}

Keywords: Legg-Perthes Disease. Osteonecrosis. Femur Head Necrosis. Femur Head.

\section{INTRODUÇÃO}

Vários nomes têm sido relacionados com a doença de Legg-CalvéPerthes (DLCP): Osteocondrite dissecante da cabeça femoral, necrose avascular da cabeça femoral, osteocondrite, coxae juvenilis, coxa plana, necrose asséptica, osteose idiopática. (WARREN; DINGWALL, 1972; FOSSUM, 2002). 
SANTANA FILHO, M.V. et al. Doença de Legg-Calvé-Perthes. Revisão bibliográfica. PUBVET, Londrina, V. 5, N. 9, Ed. 156, Art. 1052, 2011.

A DLCP é uma necrose asséptica avascular que resulta em uma deformidade na superfície articular. É peculiar de cães de pequeno porte entre 4 e 12 meses de idade ocorrendo antes do fechamento da fise da cabeça femoral. A incidência está estimada de 2 a $5 \%$ com igual distribuição entre os sexos. Caracteriza-se clinicamente por claudicação, dor, inabilidade para sustentar o peso, atrofia por desuso dos tecidos moles e diminuição da função da articulação. DLCP é relatada como bilateral entre 12-16 \% dos casos. (LJUNGGREN，1967; LEE; FRY， 1969; WARREN; DINGWALL， 1972; GAMBERDELLA，1981; NUNAMAKER， 1985; FOSSUM， 2002; DEMKO; MCLAUGHLIN, 2005).

Descrita pela primeira vez em 1910 pelo norte-americano Legg, o francês Calvé e o alemão Perthes, de maneira independente, essa afecção de etiologia ainda desconhecida é definida como sendo auto limitante e caracterizada pela necrose avascular idiopática da cabeça femoral, parcial ou total, com suas complicações associadas, que acomete crianças não-portadoras de outras doenças com idade inferior a 4-5 anos. Ocorre mais frequentemente no sexo masculino, em uma proporção de 4:1, na faixa dos dois aos 12 anos, sendo bilateral em cerca de $10 \%$ dos casos. A incidência na população varia de 1:1200 a 1:12000 e depende da localização geográfica, estando aumentada entre japoneses, mongóis, esquimós e europeus; a doença de Legg-CalvéPerthes é tida como rara em negros, nativos australianos e polinésios, e índios americanos. O processo é autolimitado, torna a cabeça do fêmur e quadril deformada com uma gama limitada de movimento e de riscos futuros para as alterações secundárias artríticas. (CAFFEY, 1973; LAREDO FILHO et al., 2001; MAMAN et al., 2007).

As mudanças histológicas e patogênicas foram descritas por Lee e Fry em 1969 e Lee em 1970. (ROBINSON, 1992)

O primeiro relato na literatura em Medicina Veterinária foi feita por Tutt em 1935. Legg, Calvé e Perthes descreveram a entidade clínica, porém somente Legg declarou a mais aceitável patogênese, no qual acreditou ser um defeito no suprimento de sangue da epífise femoral. Calvé acreditava que a 
SANTANA FILHO, M.V. et al. Doença de Legg-Calvé-Perthes. Revisão bibliográfica. PUBVET, Londrina, V. 5, N. 9, Ed. 156, Art. 1052, 2011.

condição deveria ser por uma ricketsia, e Perthes relacionou a doença a uma artrite degenerativa, provavelmente de natureza infecciosa. (NUNAMAKER, 1985)

\section{REVISÃO DE LITERATURA}

\subsection{ETIOLOGIA}

A etiologia é desconhecida, mas um número de causas têm sido apresentadas como predisposição para a doença. Uma elevação na pressão do fluido sinovial por uma reação (por exemplo trauma ou inflamação) pode ocluir o suprimento sanguíneo para a cabeça femoral com resultante necrose avascular, particularmente em cães com um anormal suprimento sanguíneo da cabeça femoral. (DINGWALL; WARREN, 1972; DEMKO; McLAUGHLIN, 2005).

Em humanos, a doença é uma afecção de etiologia desconhecida e definida como autolimitante. (CATTERALL, 1971; LAREEDO FILHO, 2001).

Legg em sua tese original sugeriu que o problema vascular era devido a um trauma, mas Trueta, descrevendo em seu trabalho a "anemia epifiseal" enfatiza o precário suprimento sanguíneo da epífise femoral de crianças e que esse suprimento sanguíneo incrementa enquanto a criança se desenvolve. $O$ suprimento sanguíneo da cabeça femoral de cães imaturos parece mais completo como sugerido por Bassettand e colaboradores (1966). Esta múltipla origem da rede vascular da cabeça do fêmur nos cães permite espaço para adicionais hipóteses (figura 1). (NUNAMAKER, 1985) 
SANTANA FILHO, M.V. et al. Doença de Legg-Calvé-Perthes. Revisão bibliográfica. PUBVET, Londrina, V. 5, N. 9, Ed. 156, Art. 1052, 2011.

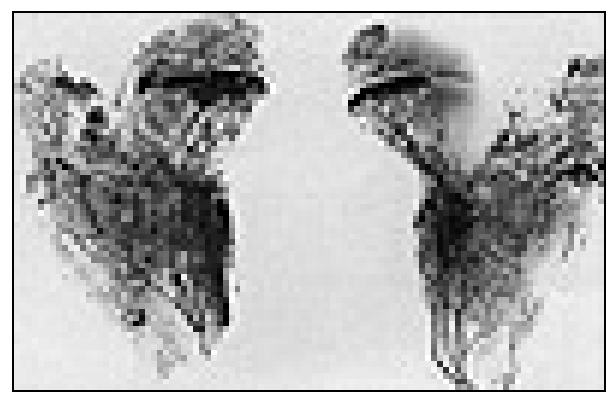

Figura 1 - Padrão vascular normal do fêmur proximal de um cão jovem (esquerda) comparado com um no qual somente os vasos reticulares inferiores estão intactos (direita). (NUNAMAKER, 1985)

A condição pode ser atribuída também por precoce atividade hormonal com prematuro fechamento da placa epifiseal e excessiva formação de osso endosteal com interferências no suprimento sanguíneo. (DINGWALL; WARREN, 1972)

Ljunggren (1966) sugestionou uma possível etiologia endócrina e mostrou a osteonecrose que ocorre com uma alta dosagem de estreróides (estrógeno e/ou testosterona).

\subsection{INCIDÊNCIA}

Pidduck (1978) analisando o pedigree de cães em um canil de poodles toy sugeriu a hipótese de que a DLCP é causada por homozigose de um gene recessivo autossômico.

Cães afetados clinicamente não devem ser usados para reprodução, ou pelo, os cruzamentos que produziram tais cães não devem ser repetidos. (DENNY; BUTTERWORTH, 2006).

Vallin (1986) indicou hereditariedade da doença na raça West Highland White Terrier.

DLCP é uma condição herdade em Manchester Terries. (VASSEUR, 1989). 
SANTANA FILHO, M.V. et al. Doença de Legg-Calvé-Perthes. Revisão bibliográfica. PUBVET, Londrina, V. 5, N. 9, Ed. 156, Art. 1052, 2011.

Robinson (1992) estudou a incidência de DLCP em 4 pequenas raças, entre elas o West Higland White Terrier e o Yorkshire Terrier. Propôs que a condição é um percentual onde a incidência é coincidente com a freqüência esperada do gene autossomal recessivo. O símbolo proposto para o gene é pd

LaFond et al. (2002) em um estudo epidemiológico para determinar o risco para desenvolver doenças ortopédicas conduzido com 300.122 novos casos do Veterinary Medical Database da Universidade de Purdue durante o período de 1986 a 1995 demonstrou que as raças com o maior risco de desenvolver DLCP entre outras doenças ortopédicas são Pastor Australiano; Cairn Terrier; Chihuahua; Dachshund; Lhasa apso; Pinscher miniatura; Pug; Poodle Toy; West Highland White Terrier; Yorkshire Terrier.

\subsection{PATOGENIA}

O centro de crescimento relacionado com a DLCP é chamado de epífise femoral principal (figura 2).

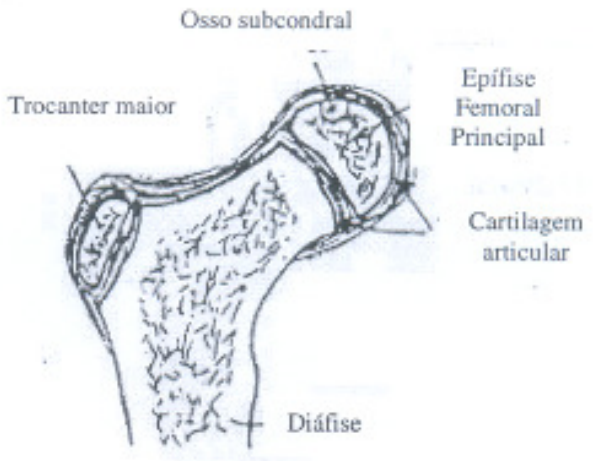

Figura 2 - O fêmur em desenvolvimento no filhote (HISLOP, 2003).

Quando essa placa de crescimento é ossificada inteiramente (aproximadamente 6-8 meses de idade nos terriers; muito mais tarde em raças grandes) dar-se-à forma a cabeça esférica do fêmur (figura 3) e solda-se com o colo e o eixo do osso. (HISLOP, 2003) 
SANTANA FILHO, M.V. et al. Doença de Legg-Calvé-Perthes. Revisão bibliográfica. PUBVET, Londrina, V. 5, N. 9, Ed. 156, Art. 1052, 2011.
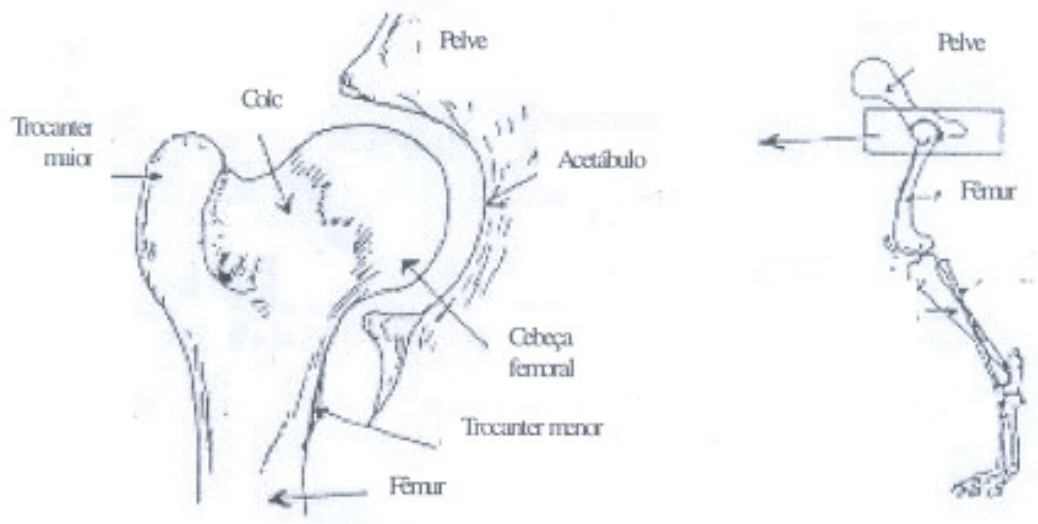

Figura 3 - Articulação normal da coxa no animal adulto (HISLOP, 2003).

Há um número de patologias que afetam os centros preliminares e secundários da ossificação que, como um grupo, são chamados de osteocondrose. A DLCP cai nesta classificação. A maioria dos autores concorda que a primeira mudança que ocorre na articulação da coxa é um episódio isquêmico, algum evento que interrompe a fonte de sangue da epífise femoral principal (figura 4).

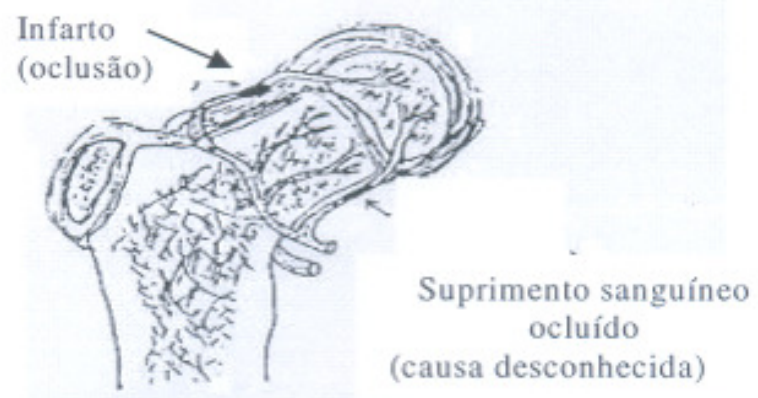

Figura 4 - Oclusão do suprimento sangüíneo causando isquemia na epífise, sendo esta condição avascular para o crescimento do osso (HISLOP, 2003).

Se a fonte de sangue é ocluída o crescimento do osso cessa, temporariamente. Ao mesmo tempo a cartilagem sobre essa junção continua a crescer porque é nutrida pelo líquido sinovial. Esta disparidade entre o 
SANTANA FILHO, M.V. et al. Doença de Legg-Calvé-Perthes. Revisão bibliográfica. PUBVET, Londrina, V. 5, N. 9, Ed. 156, Art. 1052, 2011.

crescimento do osso e da cartilagem cria um espaço articular e um pequeno centro de ossificação, o qual pode ser detectado em uma placa de raios-X. (HISLOP, 2003).

Depois da oclusão dos vasos sanguíneos, novos capilares irão surgir para restaurar a circulação, mas o osso será irregular e a remoção do osso necrosado excede a reposição de um neo-osso. Como resultado, a área na porção superior na cabeça começa a fragilizar biomecanicamente. A partir daí, a doença é subclínica, e o filhote não tem sintomas. A fraqueza biomecânica da cabeça femoral pode vir a melhorar e o crescimento normal se restabelecer. Nessa circunstância, o filhote irá ficar assintomático e com boa taxa de mobilidade na articulação da coxa. (HISLOP, 2003)

A forma sintomática da DLCP ocorre quando o osso enfraquecido da cabeça do fêmur fratura (figura 5). A maioria das opiniões inclina-se para a ocorrência da fratura após exercícios, uma injúria facilmente identificável. O animal começa a sentir dor a partir da fratura e desta forma dar-se o início da DLCP clínico. (HISLOP, 2003)

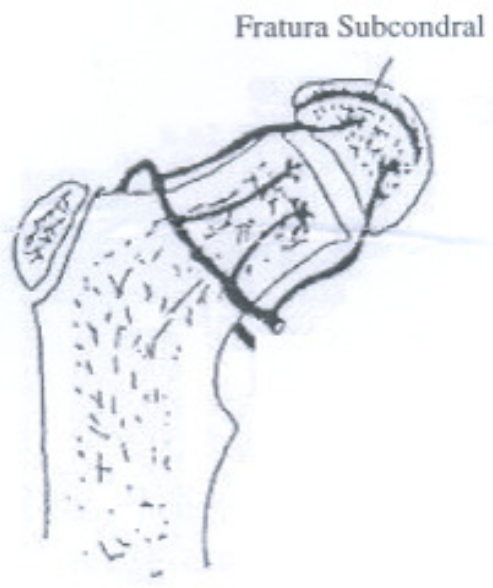

Figura 5 - Fratura do osso subcondral, pode ser pequena ou se estender por toda área da epífise (HISLOP, 2003). 
SANTANA FILHO, M.V. et al. Doença de Legg-Calvé-Perthes. Revisão bibliográfica. PUBVET, Londrina, V. 5, N. 9, Ed. 156, Art. 1052, 2011.

Uma intermitente claudicação unilateral é frequentemente o primeiro sinal da progressão da doença, mas alguns casos são totalmente assintomáticos. Uma mudança no temperamento como agressividade e relutância para ser tocado ou um precoce comportamento sexual podem ser também aparentes. Um completo exame físico e radiológico deve ser conduzido para eliminar a possibilidade de: flap da epífise femoral, fratura de colo femoral, luxação da coxa e da patela. (DINGWALL; WARREN, 1972)

O exame macroscópico da cabeça femoral de cães afetados revela que:

1. Em casos recentes existe hiperemia da articulação e dos tecidos ao redor; o líquido sinovial é límpido e transparente;

2 Em casos de longa duração a cápsula articular é engrossada, a superfície articular torna-se rugosa e irregular, e pode estar aumentada a viscosidade do líquido sinovial;

3 Em casos avançados a cabeça femoral e a superfície articular estão grossamente aplanadas e deformadas e a osteoartrite está presente. (DINWALL; WARREN, 1972)

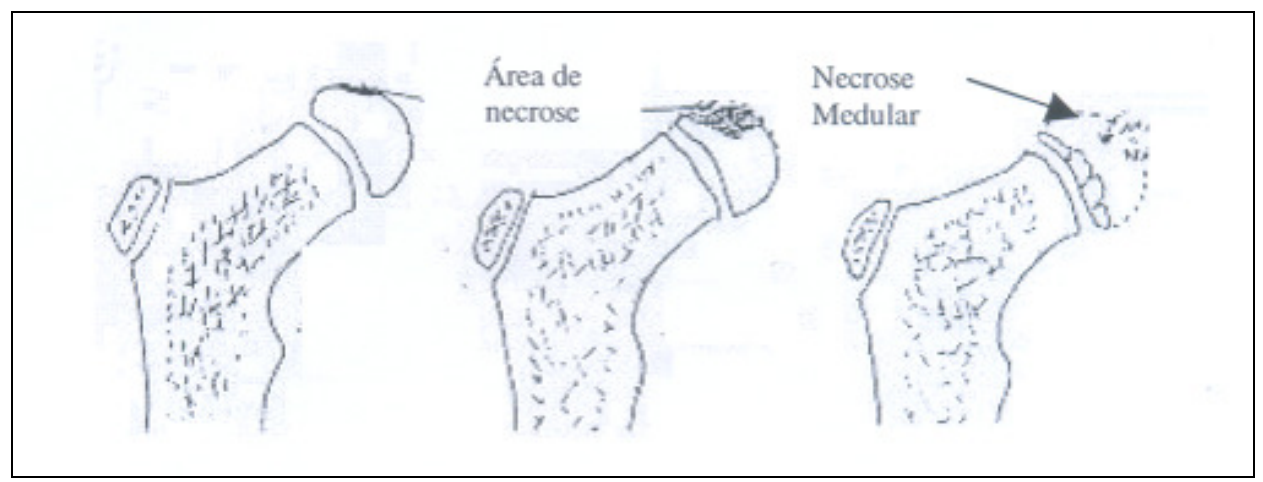

Figura 6 - Reabsorção óssea (necrose) na DLCP. (HISLOP, 2003). 
SANTANA FILHO, M.V. et al. Doença de Legg-Calvé-Perthes. Revisão bibliográfica. PUBVET, Londrina, V. 5, N. 9, Ed. 156, Art. 1052, 2011.

\subsection{SINAIS CLÍNICOS}

O animal com DLCP usualmente apresenta claudicação. Ao exame clínico mostra alguma dor na extensão da articulação da coxa, particularmente com rotação interna. O cão também irá evidenciar dor na abdução forçada da articulação coxofemoral. Trauma recente é relatado. Sinais avançados incluem contratura muscular e/ou aparente diminuição do lado afetado associado com o colapso da cabeça femoral. (figura 7). (JUNGGREN, 1967; LEE; FRY, 1969; DINGWALL; WARREN，1972; NUNAMAKER，1985; DEMKO; MCLAUGHLIN， 2005; DENNY; BUTTERWORTH, 2006).
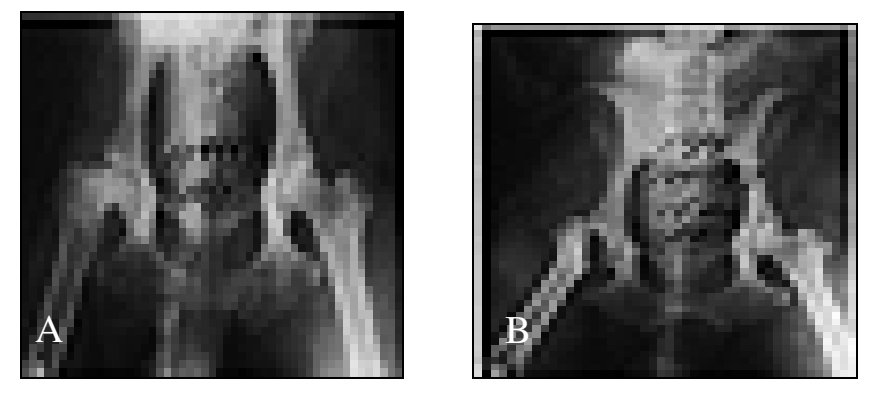

Figura 7 - (A) Radiografia crânio-caudal demonstrando o colapso da cabeça femoral em um cão com a doença de Legg-Calvé-Perthes; (B) A articulação coxofemoral após 14 meses de ressecção da cabeça e colo femorais (NUNAMAKER, 1985).

\subsection{DIAGNóSTICO}

O diagnóstico da DLCP é baseado no histórico, exame físico, achados radiográficos e histopatológicos. (DEMKO; McLAUGHLIN, 2005). Ao se examinar os pacientes acometidos pela DLCP, é importante afastar outras doenças ortopédicas que podem causar sintomas similares, como o deslizamento da epífise femoral proximal ou luxação de patela. (DENNY; BUTTERWORTH, 2006). 
SANTANA FILHO, M.V. et al. Doença de Legg-Calvé-Perthes. Revisão bibliográfica. PUBVET, Londrina, V. 5, N. 9, Ed. 156, Art. 1052, 2011.

\subsubsection{DIAGNÓSTICO RADIOGRÁFICO}

As alterações ósseas iniciais, na necrose asséptica idiopática no cão, não são demonstradas radiograficamente, são limitadas a um tênue aumento da densidade óssea da epífise femoral afetada. Um espessamento trabecular, e a formação de osso subcondral compacto continuam, até que haja evidencia de um leve aumento da densidade da cabeça femoral. (KEALY; McALLISTER, 2000; STURION; STURION, 2000).

A velocidade com que essas alterações aparecerão é imprevisível, uma vez que o cão primeiramente mostrará os sinais clínicos. Se as radiografias forem realizadas por ocasião da primeira consulta, as alterações poderão variar de praticamente imperceptíveis até bem avançadas, dependendo da vigilância do proprietário e do que as alterações realmente causam na articulação para se tornarem dolorosas aquele paciente (DENNY; BUTTERWORTH, 2006).

Os primeiros sinais radiográficos são aqueles de aumento de espaço articular. Sinais radiográficos na cabeça femoral por si só são somente evidenciados depois que começa a reabsorção vascular da necrose do osso. Esta é a hora que começa o perigo do colapso da cabeça femoral. Se deixar seguir seu curso normal, irá ocorrer colapso da cabeça femoral com destruição da articulação coxofemoral, seguido por fragmentação da cabeça femoral. Estas mudanças na estrutura resultam em uma significante perda da função com secundária atrofia muscular (figura 8). (NUNAMAKER, 1985)

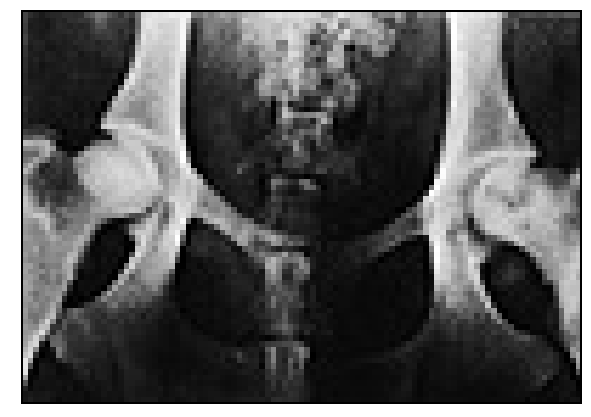

Figura 8 - Radiografia mostrando a reabsorção da cabeça femoral. (NUNAMAKER, 1985). 
SANTANA FILHO, M.V. et al. Doença de Legg-Calvé-Perthes. Revisão bibliográfica. PUBVET, Londrina, V. 5, N. 9, Ed. 156, Art. 1052, 2011.

Radiograficamente, a doença tem sido classificada em cinco graus. As apresentações radiográficas são:

1. Áreas de diminuição da densidade perto da linha da epífise em casos recentes.

2. Um aumento na largura do espaço da articulação é visualizado no início do curso da doença e essa anormalidade persiste.

3. O aspecto antero-lateral do acetábulo freqüentemente pode ter produção inicial de osteófitos tornando-se mais pronunciado como a condição persistir.

4. Um achatamento e/ou irregularidade da superfície articular da cabeça femoral é um achado comum. Vários graus podem ser vistos desde mínima perda do contorno do aspecto antero-dorsal aspecto da superfície articular a marcada depressão côncava do aspecto antero-dorsal e perda do contorno da superfície articular.

5. Fragmentação da cabeça femoral. (DINGWALL; WARREN, 1972) 
SANTANA FILHO, M.V. et al. Doença de Legg-Calvé-Perthes. Revisão bibliográfica. PUBVET, Londrina, V. 5, N. 9, Ed. 156, Art. 1052, 2011.

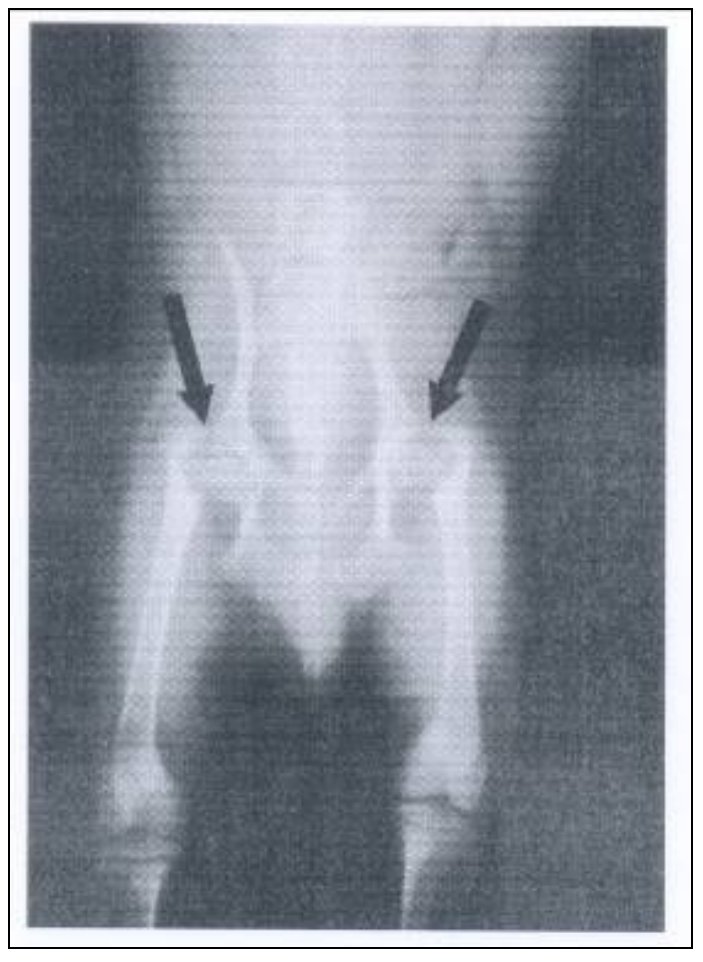

Figura 9 - Radiografia ventrodorsal demonstrado articualções coxofemorais de um cão com DLCP. Colapso bilateral da cabeça femoral (setas) (DEMKO; MCLAUGHLIN, 2005). 
SANTANA FILHO, M.V. et al. Doença de Legg-Calvé-Perthes. Revisão bibliográfica. PUBVET, Londrina, V. 5, N. 9, Ed. 156, Art. 1052, 2011.

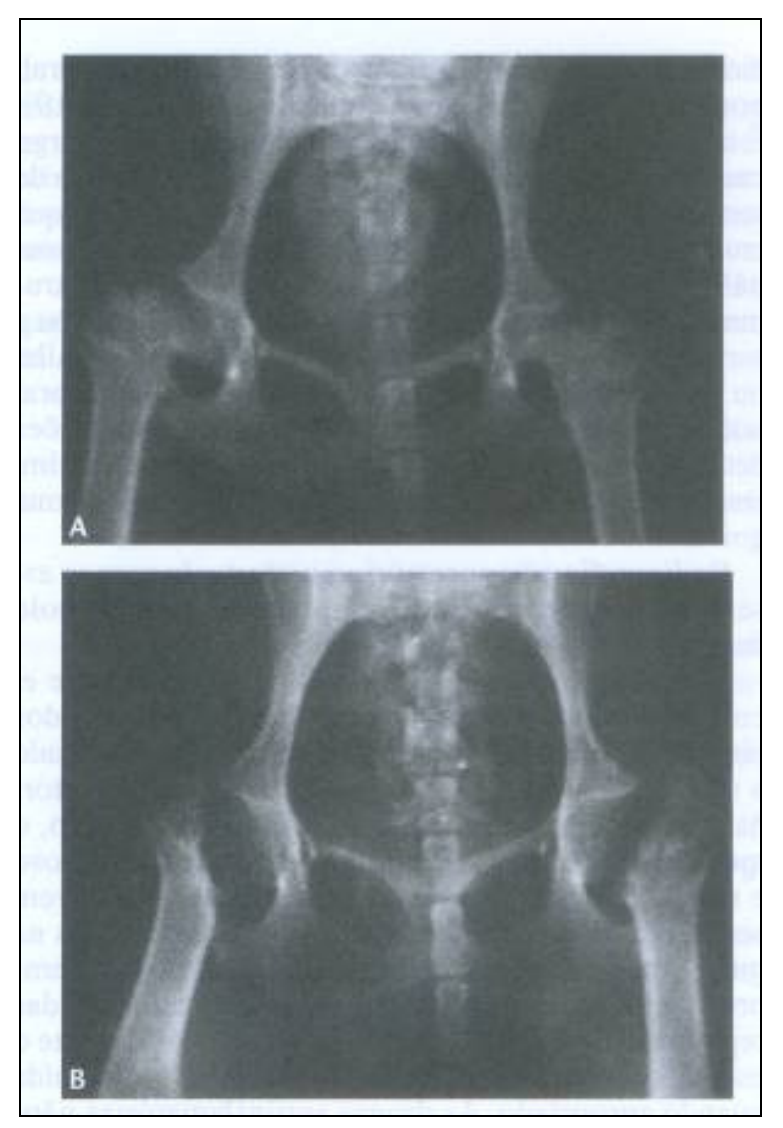

Figura 10 - (A) radiografia ventrodorsal da pelve de um West Highland Terrier de 14 meses de idade, mostrando deformidade de ambas cabeças femorais, aumento dos espaços articulares e preenchimento dos acetábulos. O cão tinha apresentado claudicação no membro pélvico direito quando filhote e agora claudicava intermitentemente de ambos os membros pélvicos. Esses achados radiográficos são considerados patognomônicos para a doença de LeggPerthes. (B) Radiografia ventrodorsal da mesma pelve, 6 semanas após a artroplastia excisional, no quadril esquerdo, e pós-operatório imediato no quadril direito. A ressecção da cabeça e do colo femoral bilateralmente é adequada. (DENNY; BUTTERWORTH, 2006) 
SANTANA FILHO, M.V. et al. Doença de Legg-Calvé-Perthes. Revisão bibliográfica. PUBVET, Londrina, V. 5, N. 9, Ed. 156, Art. 1052, 2011.

\subsubsection{DIAGNÓSTICO HISTOPATOLÓgICO}

Roperto et al. (1992), analisando 14 cães com DLCP com eletromicroscopia verificou em todos os casos eram caracterizados por mudanças necróticas polimórficas que envolve principalmente o núcleo epifiseal e o tecido ósseo metafiseal. Nesses locais, algumas vezes era possível documentar a coexistência de espaços medulares hipercelulares e áreas que foram aumentadas devido a erosões trabeculares. A presença de zonas largas originadas de completa destruição trabecular também foi documentada. Nestas zonas, debris necróticos amorfos associados com eritrócitos extravasculares e resto de tecido ósseo algumas vezes foram encontrados.

Lee (1970) em um estudo radiográfico de trinta e seis casos de DLCP e de mudanças histológicas de vinte e seis esses casos descreveu uma variedade de mudanças afetando a metáfise proximal, epífise e acetábulo.

A cabeça femoral excisada foi fixada em formol-salina, descalcificada, e embebida em cera de parafina. Seç̧ões foram obtidas no plano sagital e coradas com haematoxilina e eosina, Van Gieson e Toluidene azul. A aparência histológica foi estudada na secção representativa de cada espécime, e as seguintes características foram observadas: (figura 9) (Lee, 1970).

- A presença de trabéculas ósseas necróticas e tecido medular;

- Presença de tecido de granulação vascular invasivo;

- O grau de deformidade da superfície articular da cabeça femoral;

- A presença e extensão de rachaduras, ou cavidades, imediatamente abaixo do osso subcondral;

- Áreas densas, irregulares de osso trabecular e focos de tecido fibroso ou fibrocartilaginoso. 
SANTANA FILHO, M.V. et al. Doença de Legg-Calvé-Perthes. Revisão bibliográfica. PUBVET, Londrina, V. 5, N. 9, Ed. 156, Art. 1052, 2011.

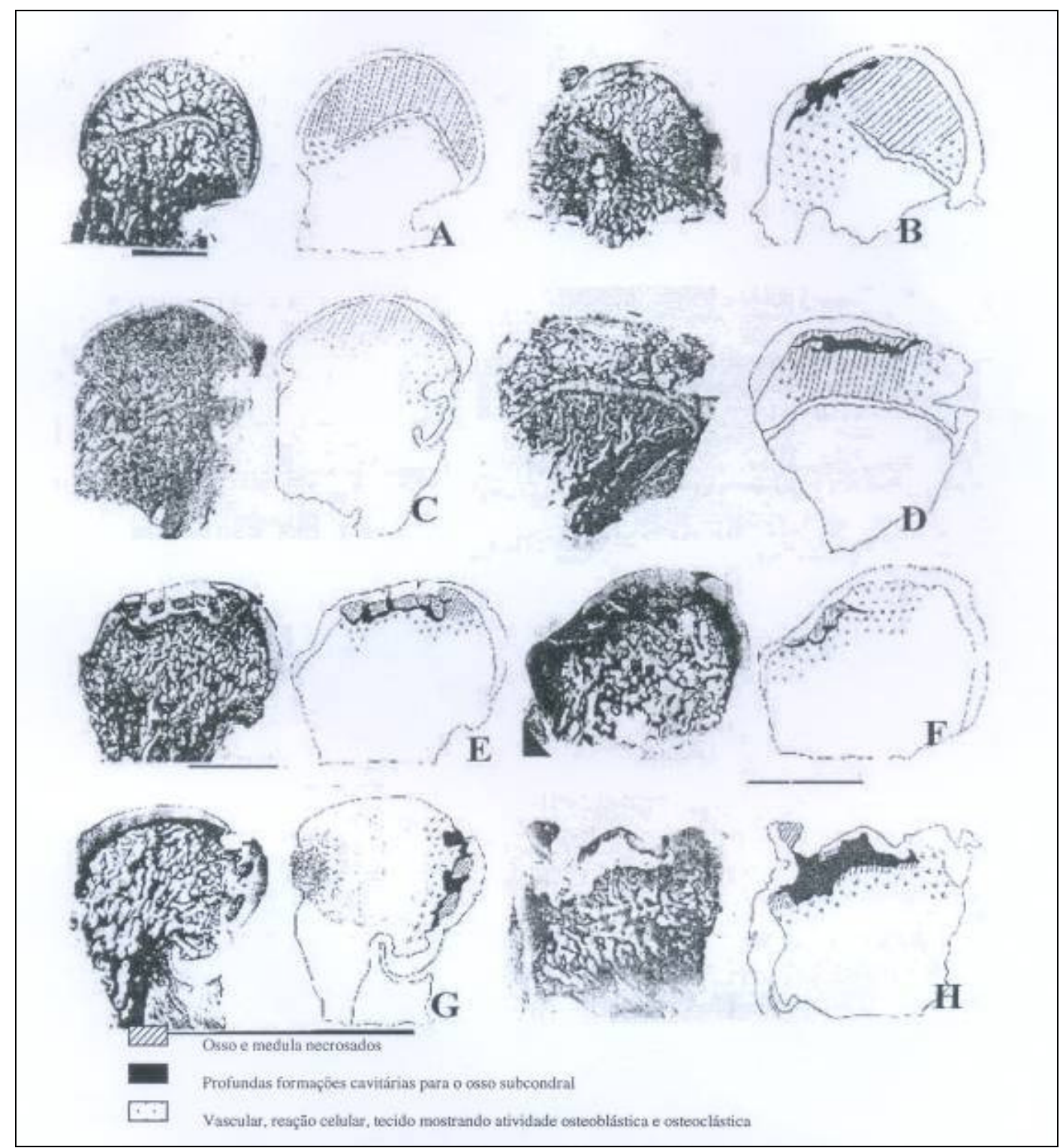

Figura 11 - Cortes histológicos da cabeça do fêmur. (A) Poodle, fêmea, claudicação por 1 semana antes da excisão da cabeça femoral direita. (B) Yorkshire Terrier, fêmea, claudicação por 3 semanas antes da excisão da cabeça femoral. (C) Poodle, macho, claudicação por 2 meses antes da excisão da cabeça femoral. (D) West Highland White Terrier, macho, claudicação por 2 meses e meio antes da excisão da cabeça femoral. (F) Poodle, macho, claudicação por 4 meses antes da excisão da cabeça femoral. (G) West Highland White Terrier, fêmea, claudicação por 5 meses antes da excisão da cabeça femoral. (H) Cairn Terrier, fêmea, claudicação por 6 meses antes da excisão da cabeça femoral. (Lee, 1970) 
SANTANA FILHO, M.V. et al. Doença de Legg-Calvé-Perthes. Revisão bibliográfica. PUBVET, Londrina, V. 5, N. 9, Ed. 156, Art. 1052, 2011.

\subsection{TRATAMENTO}

O tratamento conservador e cirúrgico tem sido usado para aliviar os sinais de dor e a claudicação provocada pela DLCP. O tratamento conservador tem incluído restrição ao exercício por 4 a 8 semanas para substituição das áreas isquêmicas por tecidos de granulação e as áreas subjacentes sejam remodeladas para acomodar a tensão alterada sobre a cabeça do fêmur, suplementação vitamínica, tratamento dietético, analgésicos e antiinflamatórios, embora os resultados frequentemente tem sido insatisfatórios. Vários estudos reportam a interrupção da claudicação em menos de 25\% dos cães tratados conservativamente (LUUNGGREN, 1967). Segundo esses estudos, a escolha do tratamento depende da severidade da doença e da progressão avaliada clínica e radiologicamente. (GIBSON et al., 1990; DEMKO; McLAUGHLIN, 2005; DENNY; BUTTERWORTH, 2006).

O tratamento cirúrgico recomendado, excisão da cabeça e colo femoral. É efetivo na aparente indução de uma pseudoartrose sem dor. A excisão da cabeça do colo femoral é usada comumente na medicina veterinária para o tratamento de lesões coxofemorais, dentre essas, DLCP. Pesquisas demonstram resultados insatisfatórios em cães de grande porte. O contato osso com osso e a produção de osteófitos tem sido sugeridos como possível causa para os maus resultados. Músculos têm sido interpostos entre o fêmur e - acetábulo para reduzir o contato ósseo com resultados favoráveis. A transferência de um "flap" do bíceps femoral previne o contato osso com osso, mas é traumática, a transferência do músculo glúteo profundo é menos traumático, mas promove menos acolchoamento do tecido mole. A técnica de ressecção em cunha foi proposta em 1979 ela inclui remover a cabeça, colo e uma adjacente cunha da metáfise (figura 10). O grande trocanter é reconectado para a metáfise remanescente, isso minimiza a quantidade de exposição, cancelando adjacências ósseas para o acetábulo e facilitando o fechamento do espaço morto com justaposição da cápsula articular e músculo. (MONTGOMERY et al., 1987) 
SANTANA FILHO, M.V. et al. Doença de Legg-Calvé-Perthes. Revisão bibliográfica. PUBVET, Londrina, V. 5, N. 9, Ed. 156, Art. 1052, 2011.

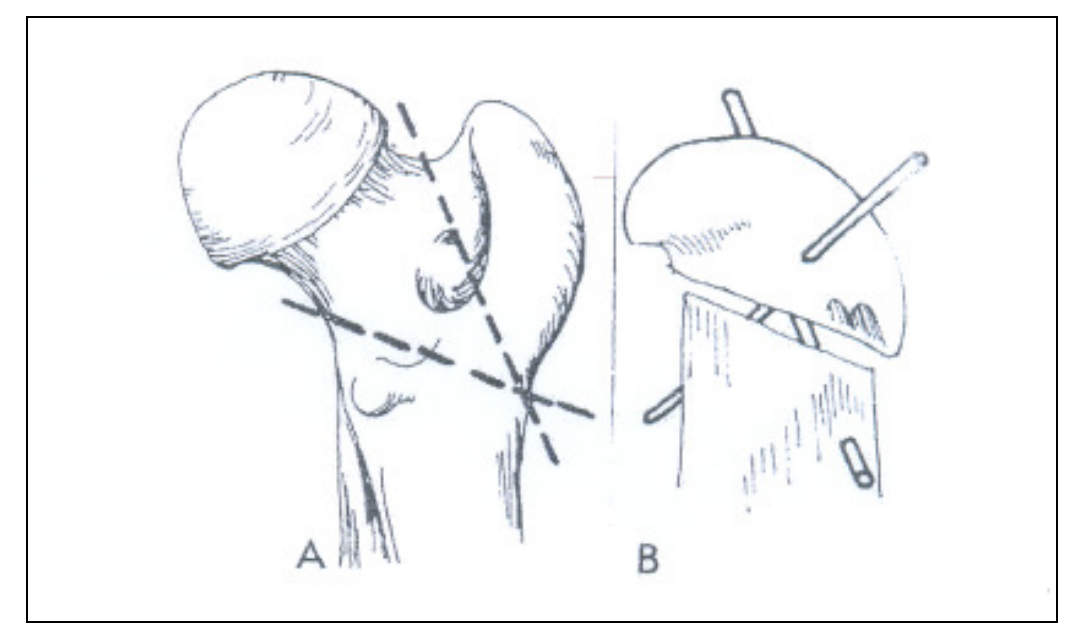

Figura 12 - Técnica de ressecção em cunha para excisão do colo e cabeça femoral. (A) Osteotomia do trocanter maior é seguida por uma osteotomia para remover a cabeça, o colo e uma cunha adjacente do osso metadiseal (linhas pontilhadas). (B) Fixação do trocanter maior para a metáfise com pinos cruzados. (MONTGOMERY et al., 1987).

Cuidados devem ser tomados na excisão da cabeça e colo femorais para não danificar o nervo ciático o qual passa caudalmente a articulação coxofemoral. Deve ser notado que se o colo femoral for seccionado transversalmente ao diâmetro, como na figura (11 a) uma projeção cônica é deixada no aspecto medial do fêmur, esta secção deve ser feita paralela a borda, como na figura (11 b). (LEE; FRY, 1969) 
SANTANA FILHO, M.V. et al. Doença de Legg-Calvé-Perthes. Revisão bibliográfica. PUBVET, Londrina, V. 5, N. 9, Ed. 156, Art. 1052, 2011.

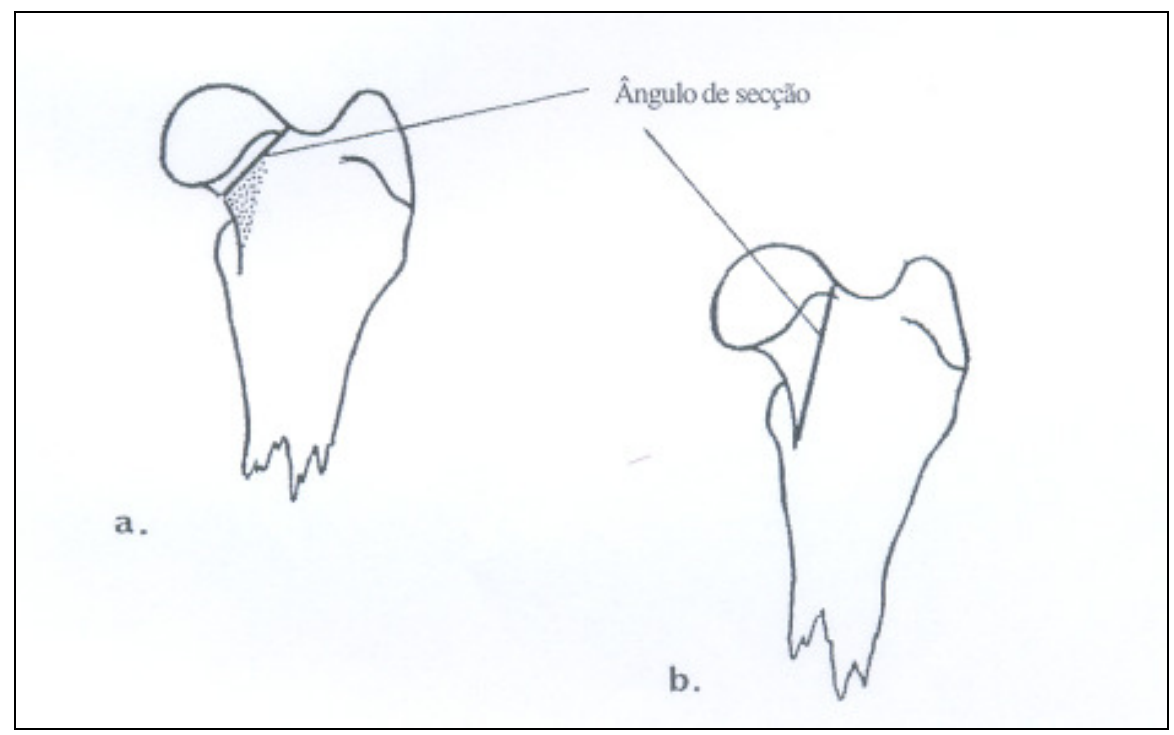

Figura 13 - Diagrama da epífise femoral. (A) Incorreto e (B) Correto ângulo pelo qual o colo femoral deve ser seccionado (LEE; FRY, 1969).

O tratamento preferido da DLCP é a artroplastia excisional da cabeça e colo femoral, sendo a abordagem craniolateral a mais realizada (DENNY; BUTTERWORTH，2006; DEMKO; McLAUGHLIN, 2005; GIBSON et al., 1990; SMITH, 1993; GAMBERDELLA, 1981). O tratamento cirúrgico melhora os sinais de dor e claudicação entre 84 e 100\% dos casos (GIBSON et al., 1990).

\section{DISCUSSÃO}

A DLCP atinge a epífise femoral e no seu curso pode chegar a destruir a cabeça e colo femoral. Muito se tem discutido sobre a etiologia da doença, mas a mais aceitável entre os autores é o bloqueio no suprimento sanguíneo para cabeça e colo femoral com reabsorção óssea e necrose asséptica. Mas Bassettand (1968) indicou que o suprimento sanguíneo de cães imaturos parece ser mais desenvolvido do que em crianças abrindo espaço a outras hipóteses. 
SANTANA FILHO, M.V. et al. Doença de Legg-Calvé-Perthes. Revisão bibliográfica. PUBVET, Londrina, V. 5, N. 9, Ed. 156, Art. 1052, 2011.

Há uma divergência entre os autores no que se refere ao início da doença. Alguns acreditam em um fator externo como um trauma, que iniciaria uma reação inflamatória sobre a articulação aumentando à pressão intraarticular e ocluiria as artérias que irrigam a cabeça femoral. Outros acreditam num fundo genético e/ou fundo endócrino onde o aumento de esteróides na corrente sanguínea levando a uma osteonecrose da epífise femoral.

A incidência é maior em cães de raças pequenas. O diagnóstico é feito através de um exame clínico completo com acompanhamento radiográfico.

O tratamento objetiva a atenuação da dor que leva a claudicação do membro afetado. O tratamento conservador não é muito utilizado, pois só apresenta sucesso em menos de $25 \%$ dos casos. O tratamento cirúrgico consiste na excisão da cabeça e colo femorais e a formação de uma pseudoartrose em substituição da articulação coxofemoral. Um dos fatores principais é o ângulo de excisão para que não se formem cunhas e se obtenha um contato osso-a-osso que leva a dor e claudicação permanente. Além do ângulo de excisão tem-se utilizado interposição de cápsula articular ou muscular entre o sítio de incisão e o acetábulo para evitar o contato osso-aosso, os músculos mais utilizados são o bíceps femoral e o glúteo profundo. 0 uso ou não de interposições vai depender do tamanho do animal e do grau de comprometimento da articulação. Em animais de pequeno porte, segundo Montgomery (1987), não se observou diferenças entre o uso de interposições.

\section{CONSIDERAÇõES FINAIS}

A Doença de Legg- Calvé-Perthes é uma osteonecrose idiopática da cabeça femoral em crianças e cães. Acomete crianças não-portadoras de outras doenças com idade inferior a 4-5 anos e cães de pequeno porte entre 4 e 12 meses de idade. A artrite se instala após a perda da integridade da superfície articular. O diagnóstico é baseado no histórico, exame físico, achados radiográficos e histopatológicos. O tratamento conservador e cirúrgico tem sido 
SANTANA FILHO, M.V. et al. Doença de Legg-Calvé-Perthes. Revisão bibliográfica. PUBVET, Londrina, V. 5, N. 9, Ed. 156, Art. 1052, 2011.

usado para aliviar os sinais de dor e claudicação.

\section{REFERÊNCIAS BIBLIOGRÁFICAS}

Bassett FH, Wilson JW, Allen BW et al: Normal vascular anatomy of the head of the femur in puppies with emphasis on the inferior retinacular vessels. J Bone Joint Surg 51:1139, 1968

Berson JL, et al. A retrospective study of the efficacy of femoral and neck excisions in 94 dogs and cats. Vet Surg 1980; 9:88.

Caffey J. Pediatric X-ray diagnosis. $6^{\text {th }}$ ed. Chicago: Year Book Medical Publisher; 1973. p.1165.

Catterall A. The natural history of Perthes disease. J Bone Joint Surg 1971; 53B:37-53.

Demko J, McLaughlin R. Developmental orthopedic disease. Vet Clin North Am Small Anim Pract 2005; 35(5):1111-35.

Denny HR; Butterworth, SJ. Cirurgia ortopédica em cães e gatos. 4th ed. São Paulo: Roca. P. 362-64.

Fossum TW. Cirurgia de Pequenos Animais. 1st ed. São Paulo: Roca; 2002. p. 941-1042.

Gambardella PC. Legg-Calvé-Perthes disease in dogs. In: Bojrab MJ, editor. Disease mechanisms in small animal surgery. $2^{\text {nd }}$ ed. Philadelphia: Lippincott Williams \& Wilkins; 1993. p. 804-7.

Gibson $\mathrm{KL}$, Lewis $\mathrm{DD}$, pechman RD. use of external coaptation for the treatment of avascular mecrosis of the femoral head in a dog. J Am Vet Med Assoc 1990; 197(\&):868-70.

Hislop HM. Legg-Calvé-Perthes. Atjournal 2003.

Junggren GL. A comparative study of conservative and surgical treatment of Legg Perthes disease in the dog. J Am Anim Hosp Assoc 1966; 1:6-10.

LaFond E, Breur GJ, Austin CC. Breed susceptibility for developmental orthopedic disease in dogs. J Am Anim Hosp Assoc 2002; 38(5):467-77.

Laredo Filho J, Dias LS, Kuwajima SS. Doença de Legg-Calvé-Perthes. Clin Ortop 2001; 2:7190.

Ljunggren G. Legg-Perthes disease in the dog. Acta Orthop Scand 1967; 95:7-79.

Lee R, Fry PD. Some observations on the occurrence of Legg-Calvé-Perthes disease (coax plana) in the dog, and an evaluation of excision arthroplasty as a method of treatment. J Small Anim Pract 1969; 10(5):309-17.

Lee R. A study of the radiographic and histological chnages occuring in Legg-Calve-Perthes Disease (LCP) in the dog. J Small Anim Pract 1970; 11:621-38.

Kealy JK. McAllister H. Diagnostic radiology and ultrasonography of the dog and cat. $3^{\text {rd }}$ ed. Philadelphia: W.B. Saunders; 2000. p. 297. 
SANTANA FILHO, M.V. et al. Doença de Legg-Calvé-Perthes. Revisão bibliográfica. PUBVET, Londrina, V. 5, N. 9, Ed. 156, Art. 1052, 2011.

Maman E, Yaniv M, Segev E. Legg-Calve_perthes disease-literature review and updates. Harefuah 2007; 146(1):68-72.

Montegomery RD, Milton JL, Horne RD, Coble RH Jr, Williams JC. A retrospective comparison of three techniques for femoral head and neck excision in dogs. Vet surg 1987; 16(6):423-6.

Nunamaker DM. Legg-Calvé-Perthes. In: Newton CD, Nunamaker DM, editors. Textbook of small animal orthopaedics. Philadelphia: JB Lippincott; 1985. p. 949-52.

Pidduck $\mathrm{H}$, Webbon PM. The genetic control of Perthes' disease in toy poodles - a working hypothesis. J small Anim Pract 1978; 19(12):729-33.

Robinson R. Legg-Calvé-Perthes disease in dogs: genetic aetiology. J Small Anim Pract 1992; $33: 275-6$.

Roperto F, Papparella S, Crovace AA. Legg-Calvé-Perthes disease in dogs: histological and ultra structural invertigations. J Amer Anim Hosp Assoc 1992; 28:156-62.

Smith, MM. Perthes, disease. In: Slatter, $\mathrm{DH}$, editor. Textbook of small animal surgery. Philadelphia: W. B. Saunders; 1993. p. 1966-1977.

Sturion DJ, Sturion MAT. Radiologia Veterinária. Edunopar; 2000. p.634-42.

Vasseur PB, Foley P, Stevenson S, et al. Mode of inheritance of Perthes disease in Manchester terriers. Clin Orthop 1989; 244:281-92.

Wallin B. Perthes sjukdom hos West Highland White Terrier, en genetic studie. Swenk Veterinarttinding 1986; 38:114-18.

Warren DV, Dingwall JS. Legg-Perthes disease in the dog: a review. Can Vet J 1972; $13(6): 135-7$. 